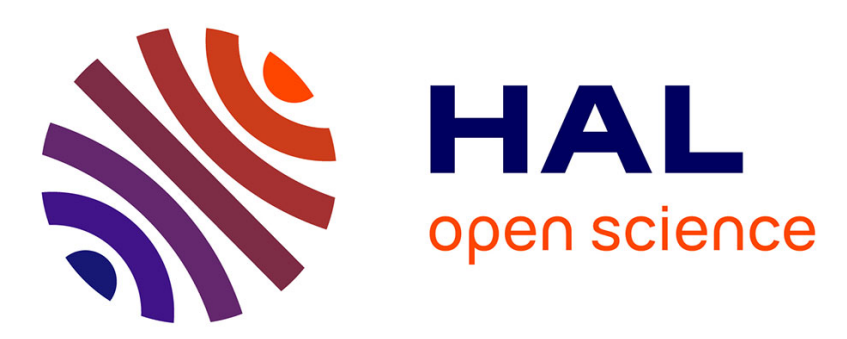

\title{
Statistically meaningful measure of domain-wall roughness in magnetic thin films
}

D. Jordán, L. J Albornoz, J. Gorchon, Charles-Henri Lambert, S. Salahuddin, J. Bokor, J. Curiale, S. Bustingorry

\section{- To cite this version:}

D. Jordán, L. J Albornoz, J. Gorchon, Charles-Henri Lambert, S. Salahuddin, et al.. Statistically meaningful measure of domain-wall roughness in magnetic thin films. Physical Review B: Condensed Matter (1978-1997), 2020, 101 (18), 10.1103/PhysRevB.101.184431 . hal-02901713

\section{HAL Id: hal-02901713 \\ https://hal.univ-lorraine.fr/hal-02901713}

Submitted on 17 Jul 2020

HAL is a multi-disciplinary open access archive for the deposit and dissemination of scientific research documents, whether they are published or not. The documents may come from teaching and research institutions in France or abroad, or from public or private research centers.
L'archive ouverte pluridisciplinaire HAL, est destinée au dépôt et à la diffusion de documents scientifiques de niveau recherche, publiés ou non, émanant des établissements d'enseignement et de recherche français ou étrangers, des laboratoires publics ou privés. 


\title{
Statistically meaningful measure of domain-wall roughness in magnetic thin films
}

\author{
D. Jordán $\odot,{ }^{1}$ L. J. Albornoz $\odot,{ }^{1,2}$ J. Gorchon $\odot,{ }^{3}$ C. H. Lambert, ${ }^{4}$ S. Salahuddin, ${ }^{4}$ \\ J. Bokor $\odot,{ }^{4,5}$ J. Curiale $\odot,,^{1,2}$ and S. Bustingorry $\oplus^{2, *}$ \\ ${ }^{1}$ Instituto Balseiro, Universidad Nacional de Cuyo - CNEA, Centro Atómico Bariloche, (R8402AGP) S. C. de Bariloche, Río Negro, Argentina \\ ${ }^{2}$ Instituto de Nanociencia y Nanotecnología, CNEA-CONICET, \\ Centro Atómico Bariloche, (R8402AGP) S. C. de Bariloche, Río Negro, Argentina \\ ${ }^{3}$ Université de Lorraine, CNRS, IJL, F-54000 Nancy, France \\ ${ }^{4}$ Department of Electrical Engineering and Computer Science, University of California, Berkeley, California 94720, USA \\ ${ }^{5}$ Materials Sciences Division, Lawrence Berkeley National Laboratory, Berkeley, California 94720, USA
}

(Received 20 August 2019; revised manuscript received 31 March 2020; accepted 12 May 2020; published 28 May 2020)

\begin{abstract}
Domain walls in magnetic thin films display a complex dynamical response when subject to an external drive. It is claimed that different dynamic regimes are correlated with the domain-wall roughness, i.e., with the fluctuations of domain-wall position due to the inherent disorder in the system. Therefore, key to understanding the dynamics of domain walls is to have a statistically meaningful measure of the domain-wall roughness. Here we present a thorough study of the roughness parameters, i.e., roughness exponent and roughness amplitude, for domain walls in a ferrimagnetic $\mathrm{GdFeCo}$ thin film in the creep regime. Histograms of roughness parameters are constructed with more than 40 independent realizations under the same experimental conditions, and the average values and standard deviations are compared in different conditions. We found that the most prominent feature of the obtained distributions is their large standard deviations, which is a signature of large fluctuations. We show that even if the roughness parameters for a particular domain wall are well known, these parameters are not necessarily representative of the underlying physics of the system. In the low field limit, within the creep regime of domain-wall motion, we found the average roughness exponent and roughness amplitude to be around 0.75 and $0.45 \mu \mathrm{m}^{2}$, respectively. When an in-plane magnetic field is applied we observed that, even though the distributions are wide, changes in the mean values of roughness parameters can be identified; the roughness exponent decreasing to values around 0.72 while the roughness amplitude increases to $0.65 \mu \mathrm{m}^{2}$. Our results call for a careful consideration of statistical averaging over different domains walls when reporting roughness exponents.
\end{abstract}

DOI: 10.1103/PhysRevB.101.184431

\section{INTRODUCTION}

One possible route to the development of new recording technologies is based on thin magnetic materials [1-7]. The basic understanding of magnetization reversal mechanisms, which are dominated by domain-wall dynamics, is thus critical. In this sense, it is crucial to understand the velocity response to external drives (magnetic field or electric current). On one hand, the linear behavior at high drives needs to be tuned for the design of fast and energetically efficient magnetic memory devices. On the other hand, the nonlinear velocity-field behavior at low drives should also be considered. For example, proper recognition of the dynamic properties within the disorder dominated depinning regime helps in designing materials with low depinning fields, allowing them to reach the regime of linear velocity-field response at lower fields.

Domain wall dynamics in thin magnetic materials results from the interplay between the elastic energy of the domain wall, the intrinsic disordered energy landscape of the sample,

*sbusting@cab.cnea.gov.ar the Zeeman energy contribution due to external magnetic fields, and the thermal energy scale. As a consequence, different dynamical regimes can be observed as a function of the external drive. Even though for thin magnetic materials the natural choice as the external drive is the magnetic field, coupling of the magnetic system with different drives, such as electric currents [2,8-11], electric fields [12-15], and strain $[16,17]$, has also been investigated. Particularly, in systems with perpendicular magnetic anisotropy, the role of in-plane magnetic fields has attracted much attention [7,15,18-23].

In the low drive region, the velocity-field response is dominated by the intrinsic disorder in the sample. This disorder can be related to surface terraces or structural defects which are originated in the growth process. A remarkable fingerprint of the disorder is the existence of the depinning field $H_{d}$. Well above $H_{d}$ the domain-wall velocity grows linearly with the external field, commonly referred to as the flow regime. Below the depinning field domain-wall dynamics is strongly hindered, leading to the so-called creep regime. In this last regime, velocity-field response can be described as a stretched exponential, $\ln v \sim-H^{-\mu}$, characterized by a universal creep exponent $\mu$ depending only on dimensionality, general elastic properties of the domain wall, and disorder characteristics. In 
fact, using scaling arguments $[24,25]$ it is possible to write that

$$
\mu=\frac{2 \zeta_{\mathrm{eq}}+d-2}{2-\zeta_{\mathrm{eq}}}
$$

where $d$ is the dimensionality of the domain wall $(d=1$ for magnetic thin films). The roughness exponent $\zeta$ gives the power-law growth of transverse fluctuations of the domainwall position as a function of the longitudinal length scale $r$, i.e., along the direction defined by the mean position of the domain wall. These fluctuations can be quantified by the roughness function $B(r)$ and, as we will show below, it follows $B(r) \sim r^{2 \zeta}$. The value of the roughness exponent entering in Eq. (1) is the one obtained at equilibrium, $\zeta_{\text {eq }}$, i.e., in the zero field limit $[24,25]$. The relationship between $\mu$ and $\zeta_{\text {eq }}$ shows that there is a strong connection between domainwall dynamics at low fields and geometric fluctuations of the domain-wall position [26]. For the case of harmonic elastic one-dimensional domain walls in a short-range correlated disorder $\zeta_{\mathrm{eq}}=2 / 3$ and thus $\mu=1 / 4$.

The importance of the connection between the creep law, $\ln v \sim-H^{-1 / 4}$, and the roughness exponent $\zeta$ was early recognized in the seminal work of Lemerle et al. [27]. It was subsequently realized that, at finite applied fields, different roughness exponents characterize different length scales of domain-wall fluctuations. The length scales at which the crossover between different roughness exponents occur strongly depend on the external magnetic field $[26,28]$. As an important consequence, one has to consider in detail the experimental accessible length scales in order to interpret roughness results [29].

Though knowing the roughness exponent is necessary for a complete understanding of domain-wall dynamics, after the work of Lemerle and coworkers [27] experimental reports of roughness exponents of domain walls in magnetic thin films are scarce (see Refs. [9,29-36]). Measuring domainwall roughness exponents would require to experimentally obtain domain-wall positions and then to define and compute a proper correlation function, such as the commonly used roughness function $B(r)$, which measures displacementdisplacement correlations. Furthermore, whether the roughness exponent should be extracted from a correlation function averaged over many domain walls or as the average of individual domain walls roughness exponents has been recently theoretically addressed using numerical models for equilibrium and depinning interfaces [37]. This last study also pointed out that the broadness of the distribution of roughness exponents should be considered when reporting reliable values. Motivated by this observation, we experimentally study in this work the roughness properties of domain walls in magnetic thin films. Our study focuses on the creep regime of magnetic field driven domain walls in $\mathrm{GdFeCo}$ thin ferrimagnetic films. We report distributions for roughness parameters in the creep regime and show that they present a non-negligible broadness. Furthermore, we study how the distributions are affected by the application of an in-plane magnetic field. In order to obtain the presented results special attention is paid to the protocol used for the acquisition and processing of images and the statistical properties of roughness parameters.
The rest of the work is organized as follows. Section II comprises experimental details and the velocity-field characteristics. The protocol used to extract roughness parameters is described in detail in Sec. III. Section IV presents the statistical analysis of the data and discusses its significance. Finally, Sec. V is devoted to a summary and a discussion of the results.

\section{SAMPLE DETAILS AND DOMAIN-WALL VELOCITY}

This section is dedicated to a description of the sample and of the main methods used in the experiments, together with the velocity-field characteristics which sets the ground for roughness analysis.

\section{A. Sample and methods}

Domain-wall roughness is studied in a GdFeCo sample which comprises a $\mathrm{Ta}(5 \mathrm{~nm}) / \mathrm{Gd}_{32} \mathrm{Fe}_{61.2} \mathrm{Co}_{6.8}(10 \mathrm{~nm}) / \mathrm{Pt}(5$ $\mathrm{nm})$ trilayer deposited on a thermally oxidized silicon $\mathrm{Si} / \mathrm{SiO}_{2}(100 \mathrm{~nm})$ substrate by RF sputtering. The thickness of each layer is indicated in parenthesis. The $\mathrm{GdFeCo}$ thin film is a rare earth-transition metal (RE-TM) ferrimagnetic compound presenting a dominant perpendicular magnetic anisotropy (i.e., magnetization lies perpendicular to the film plane) with the RE and TM magnetic moments antiferromagnetically coupled. One of the key features present in this family of materials is the magnetization compensation temperature $T_{M}$. At this characteristic temperature the RE and TM antiferromagnetically-coupled magnetic moments compensate and the net magnetization of the sample vanishes. In practice, the magnetization compensation temperature can be tuned with the composition of its constituents [38]. In addition, due to the fact that the RE and TM gyromagnetic factors may differ, an angular momentum compensation temperature at a value different from the magnetization compensation temperature can be observed. This leads for instance to very high domain-wall mobility in $\mathrm{GdFeCo}$ thin layers close to the angular momentum compensation temperature [39]. As shown in the inset of Fig. 1(b), SQUID magnetometer measurements of the magnetization of the sample indicate that the magnetization compensation temperature is $T_{M} \approx 190 \mathrm{~K}$. Although we do not have a direct measure of the angular momentum compensation temperature $T_{A}$, using the strong correlation between $T_{M}$ and $T_{A}$ reported in Ref. [40] we can estimate that $T_{A} \approx 265 \mathrm{~K}$.

Domain wall velocities were computed from differential images acquired with a polar magneto-optical Kerr effect microscope, whose spatial resolution is typically around $1 \mu \mathrm{m}$. First, sample temperature was stabilized at $T=295 \mathrm{~K}$ well above both its magnetization compensation temperature $T_{M}$ and its angular momentum compensation temperature $T_{A}$. The magnetization of the sample is first saturated with an out-of-plane magnetic field $H_{z}$ in the $-z$ direction. Then a short magnetic field pulse in the opposite direction is used to nucleate magnetic domains with positive magnetization. A second pulse of intensity $H_{z}$ and duration $\Delta t$ is used to favor the nucleated domain to grow, thus resulting in the displacement of the domain wall. Images were captured after and before the application of the magnetic field pulse. By measuring the mean domain-wall displacement in the 

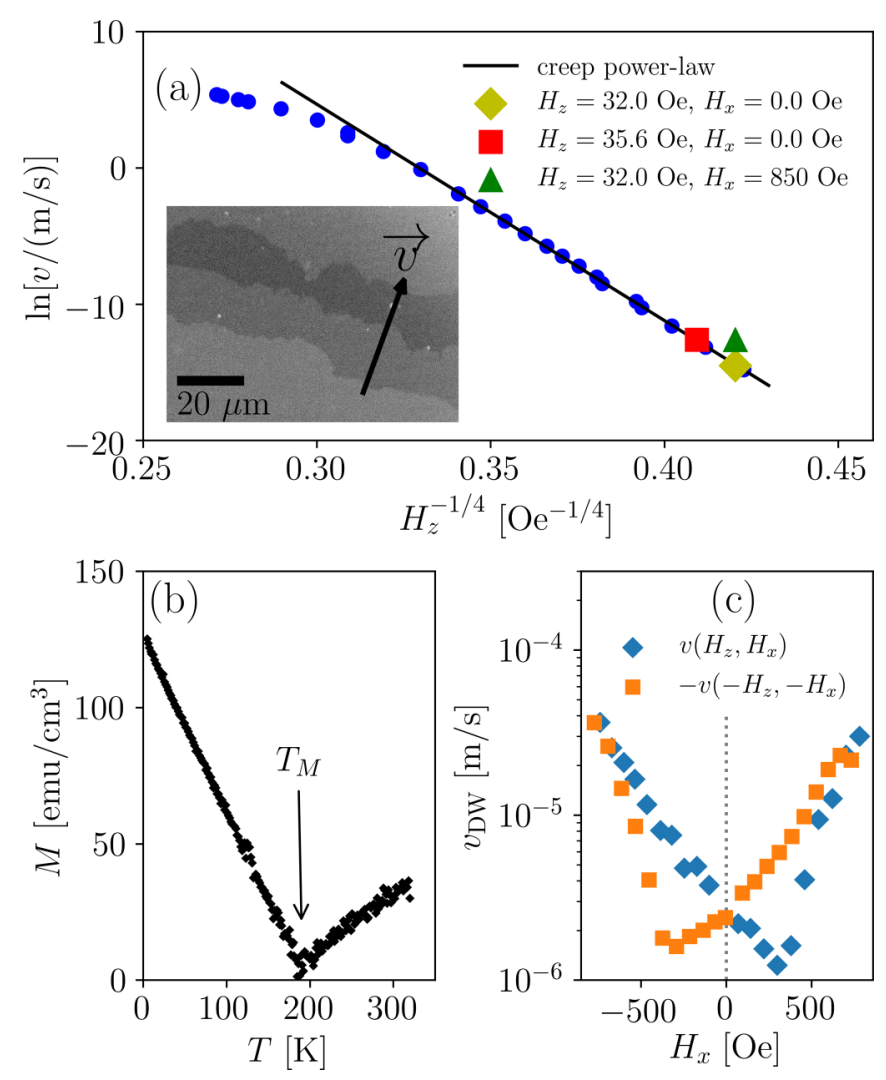

FIG. 1. (a) Creep plot, $\ln v$ against $H^{-1 / 4}$ for GdFeCo at $T=$ $295 \mathrm{~K}$. The continuous line corresponds to a linear fit in the creep regime. The diamond, square, and triangular points indicate three cases where the roughness was analyzed in detail (see main text). Inset: Domain wall displacements obtained with PMOKE microscopy. Magnetic field pulses of intensity $H_{z}=35.6$ Oe and duration $\Delta t=1 \mathrm{~s}$ where used. Different gray levels correspond to images taken every five pulses. The black arrow indicates the direction of average velocity, which is orthogonal to the mean longitudinal coordinate of the domain-wall profiles. (b) Temperature dependence of the field cooling magnetization at $H_{z}=1000 \mathrm{Oe}$, showing a magnetization compensation temperature $T_{M} \approx 190 \mathrm{~K}$. (c) Velocity as a function of the in-plane field $H_{x}$ obtained at $T=295 \mathrm{~K}$ using an out-of-plane field $H_{z}=35$ Oe.

direction perpendicular to the mean domain-wall position, $\Delta x$, between consecutive images, the mean velocity of the domain wall $v=\Delta x / \Delta t$ is obtained as a function of the outof-plane field $H_{z}$. The inset of Fig. 1(a) shows the domain-wall displacements observed when applying magnetic field pulses of intensity $H_{z}=35.6 \mathrm{Oe}$ and duration $\Delta t=1 \mathrm{~s}$. For clarity, different gray levels correspond to images taken every five pulses. Alternatively, in addition to the out-of plane field $H_{z}$, an in-plane field $H_{x}$ can be switched on by using a homemade electromagnet, which permits us to reach in-plane DC fields up to $H_{x}=2500 \mathrm{Oe}$.

\section{B. Velocity-field characteristics}

Velocity-field results are presented as a creep plot in the main panel of Fig. 1(a). Domain wall velocity-field response at fields below the depinning field $H_{d}$ is given by thermal activation over disorder energy barriers with a characteristic energy scale $k_{B} T_{d}$, with $k_{B}$ the Boltzmann constant and $T_{d}$ the depinning temperature. One thus expects for $H_{z}<H_{d}$ that

$$
v=v_{d} e^{-\Delta E /\left(k_{B} T\right)},
$$

with a depinning velocity scale $v_{d}$ and the characteristic energy barrier

$$
\Delta E=k_{B} T_{d}\left[\left(\frac{H_{z}}{H_{d}}\right)^{-\mu}-1\right],
$$

with the creep exponent $\mu=1 / 4$ (see Refs. [41,42] and references therein). Therefore, the velocity-field characteristics are shown in Fig. 1(a) as a creep plot, $\ln v$ vs $H_{z}^{-1 / 4}$. It is observed that the velocity follows the creep law (continuous line) in a wide velocity range, from $3.7 \times 10^{-7} \mathrm{~m} / \mathrm{s}$ at a field of $31.0 \mathrm{Oe}$ to $3 \mathrm{~m} / \mathrm{s}$ at $100 \mathrm{Oe}$. The fact that the velocity-field characteristic is well described by the creep law suggests that the dynamics can be described within the weak disorder limit, i.e., without strong pinning effects.

In Fig. 1(a) we also show the three cases where the roughness was analyzed in detail. The point marked as a diamond corresponds to $H_{z}=32.0 \mathrm{Oe}$ and $H_{x}=0$, a reference point in the mentioned creep regime. The point marked as a triangle corresponds to the same out-of-plane field, $H_{z}=32.0 \mathrm{Oe}$, but with a simultaneously in-plane applied field of $H_{x}=850 \mathrm{Oe}$, resulting in an increase of the domain-wall velocity. With the aim to also compare roughness at similar velocities, we studied the case with a pure out-of-plane field, $H_{z}=35.6 \mathrm{Oe}$, shown as a square shaped point in Fig. 1(a).

Application of an in-plane field $H_{x}$ results in an asymmetric velocity-field response. This can be appreciated in Fig. 1(c), where the domain-wall velocity as a function of $H_{x}$ at constant out-of-plane field $H_{z}=35 \mathrm{Oe}$ is presented. The average domain-wall longitudinal direction is vertical and the in-plane field is applied in the horizontal direction, which is the direction of displacement of the DW. The asymmetric velocity-field response shown in Fig. 1(c) is compatible with the Dzyaloshinskii-Moriya interaction [18,20,43]. Assuming the observed behavior is due to the Dzyaloshinskii-Moriya interaction, the minimum of the velocity-field curve would occur at a value $H_{\mathrm{DMI}}$ proportional to the magnitude of the Dzyaloshinskii-Moriya interaction. For planar domain-wall profiles, inverting the out-of-plane field changes the velocity direction and, if the direction of the in-plane field is also inverted, a minimum velocity at $-H_{\mathrm{DMI}}$ is obtained $[20,43]$, as shown in Fig. 1(c). The increase of the creep velocity for finite $\left|H_{x}\right|>H_{\text {DMI }}$ can be associated to a decrease of the effective energy barrier, which is proportional to the domain-wall energy density. Thus, a decrease of the effective energy barrier can be correlated with the expected decrease of the domainwall energy density with an applied in-plane field $[43,44]$. The triangular point in Fig. 1(a) was obtained at $H_{x}=850 \mathrm{Oe}$, which is almost three times the Dzyaloshinskii-Moriya field $H_{\text {DMI }} \approx 300$ Oe. As shown in Fig. 1(c), for this particular magnitude of the in-plane field the velocity does not change with the inversion of $H_{x}$ [i.e., $v\left(H_{x}\right)=v\left(-H_{x}\right)$ ]. As in a general sense the roughness of the domain wall is expected to be correlated with its velocity, this suggests that the roughness parameters should not depend on the direction of $H_{x}$ with respect to the domain-wall velocity for $\left|H_{x}\right| \approx 850$ Oe. In fact, in the results presented below, obtained with $H_{x}=850 \mathrm{Oe}$, 
we do not observe a dependence of the roughness parameters on the relative direction between domain-wall displacement and applied in-plane field. A careful analysis of the roughness parameters as a function of the relative angle between in-plane field and domain-wall displacement for different $H_{x}$ values is beyond the scope of the present work. In the following, in order to unveil the main features of a statistically representative analysis of roughness parameters we shall focus on the three cases described in Fig. 1(a).

\section{MEASURING DOMAIN-WALL ROUGHNESS}

Lets consider a domain wall whose mean position defines the longitudinal direction $r^{\prime}$, with the domain-wall position of a segment of size $L$ defined through $u\left(r^{\prime}\right)$. The roughness for a single domain wall of longitudinal size $L$ can be obtained through the roughness function $B(r)$, defined as the displacement-displacement correlation function

$$
B(r)=\frac{1}{L-r} \int_{0}^{L-r}\left[u\left(r^{\prime}+r\right)-u\left(r^{\prime}\right)\right]^{2} d r^{\prime},
$$

with $r$ lying in the longitudinal direction. Note that the normalization constant $1 /(L-r)$ properly takes into account that $L$ and $r$ might have comparable values and goes to $1 / L$ when $L \gg r$. For self-affine domain walls the roughness function is expected to grow as $B(r) \sim r^{2 \zeta}$, with $\zeta$ the roughness exponent $[45,46]$. In general, this roughness exponent characterizes the scale invariance of geometrical fluctuations of domain-wall profiles. In the context of the depinning transition, characterizing domain-wall roughness may provide information of the different dynamical regimes as a function of the external drive [26]. Domain wall profiles are therefore used to compute the roughness function, Eq. (4), which is then fit using

$$
B(r)=B_{0}\left(\frac{r}{\ell}\right)^{2 \zeta},
$$

which includes the roughness exponent $\zeta$ and the roughness amplitude $B_{0}$ as fitting parameters. The $\ell$ scale accounts for the units of the longitudinal scale $r$, so that $B_{0}$ has the same units as the roughness function $B(r)$ (here we use $\ell=1 \mu \mathrm{m}$ ). Hereafter, we shall refer to $\zeta$ and $B_{0}$ as the roughness parameters.

We shall focus our measurements of the domain-wall roughness in a low velocity range in the creep regime. In order to measure domain-wall roughness, we first locate the typical nucleation centers, then we choose a region of the sample free and far from nucleation centers. After application of nucleation and propagation magnetic field pulses we observe the appearance, in the field of view of the microscope, of an almost flat domain wall. In this case domain-wall images were captured with the magnetic field always applied. The shutter time for the camera was $\Delta t_{\text {shut }} \approx 20 \mathrm{~ms}$. Due to the small magnetic field used, the mean displacement of the domain wall during the image capture time ( $\Delta x_{\text {shut }}=v \Delta t_{\text {shut }} \approx$ $10 \mathrm{~nm}$ ) is much smaller than the resolution of the microscope and then the system is in a quasistatic condition. To confirm that, we verified that there were no differences in the results if the magnetic field was switched on or off during the image acquisition. (a)
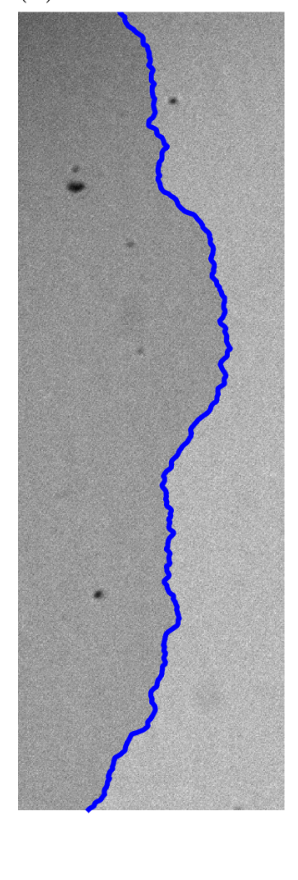

(b)

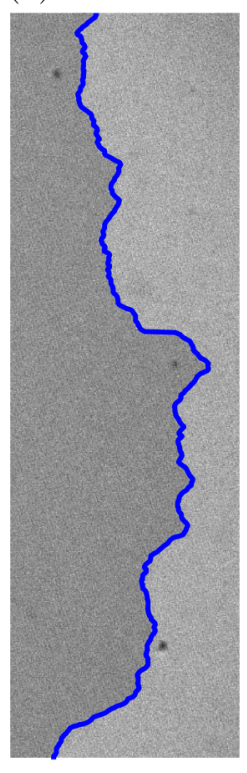

$20 \mu \mathrm{m}$ (c)

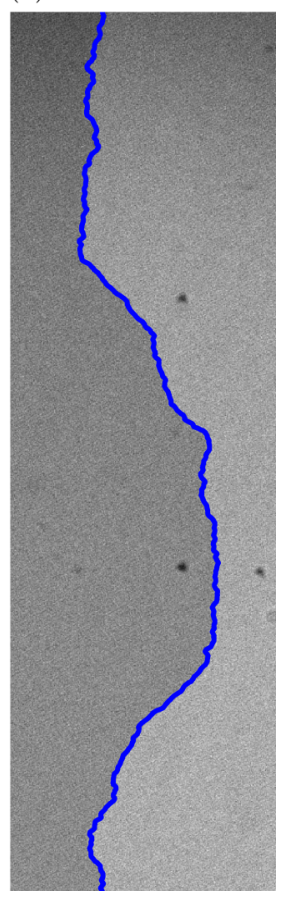

FIG. 2. Domain-wall profiles. The dark and light gray regions correspond to magnetic domains with different orientations. The blue continuous lines are the domain-wall profiles. All images were obtained with $H_{z}=35.6 \mathrm{Oe}$, with (a), (b), and (c) different realizations within the same experimental run. Domain-wall velocity in this case is $v=3.35 \mu \mathrm{m} / \mathrm{s}$. The propagation direction before the image acquisition was from left to right.

In order to illustrate how measured roughness can fluctuate between independently imaged domain walls, even under the same experimental conditions, Fig. 2 presents different domain-wall profiles obtained almost in the same sample place, with the same out-of-plane field $H_{z}=35.6 \mathrm{Oe}$ and zero in-plane field $H_{x}$, corresponding to the square point in Fig. 1(a). These images were taken starting from a given initial profile and then letting the system evolve under magnetic field pulses of intensity $H_{z}$ with the pulse duration $\Delta t$ long enough such that the positions of the domain walls before and after the application of the field pulse are uncorrelated between each other, i.e., two domain walls are not superimposed in any region, not sharing any fraction of their profiles. The external magnetic field alternates sign, $\pm H_{z}$, allowing us to obtain a large number of domain walls in the same region of the sample, thus always testing (from a statistical point of view) the same disordered energy landscape. Therefore, under this protocol we shall consider the different obtained domain-wall profiles as independent realizations under the same experimental conditions. Furthermore, domain walls images were taken in the same location of the sample for all three cases where the roughness is analyzed in detail [indicated in Fig. 1(a)], guaranteeing that the material features are always the same while the external parameters are changed. For example, the images in Fig. 2 are image crops of different domain walls obtained within the same field of view of the microscope. 

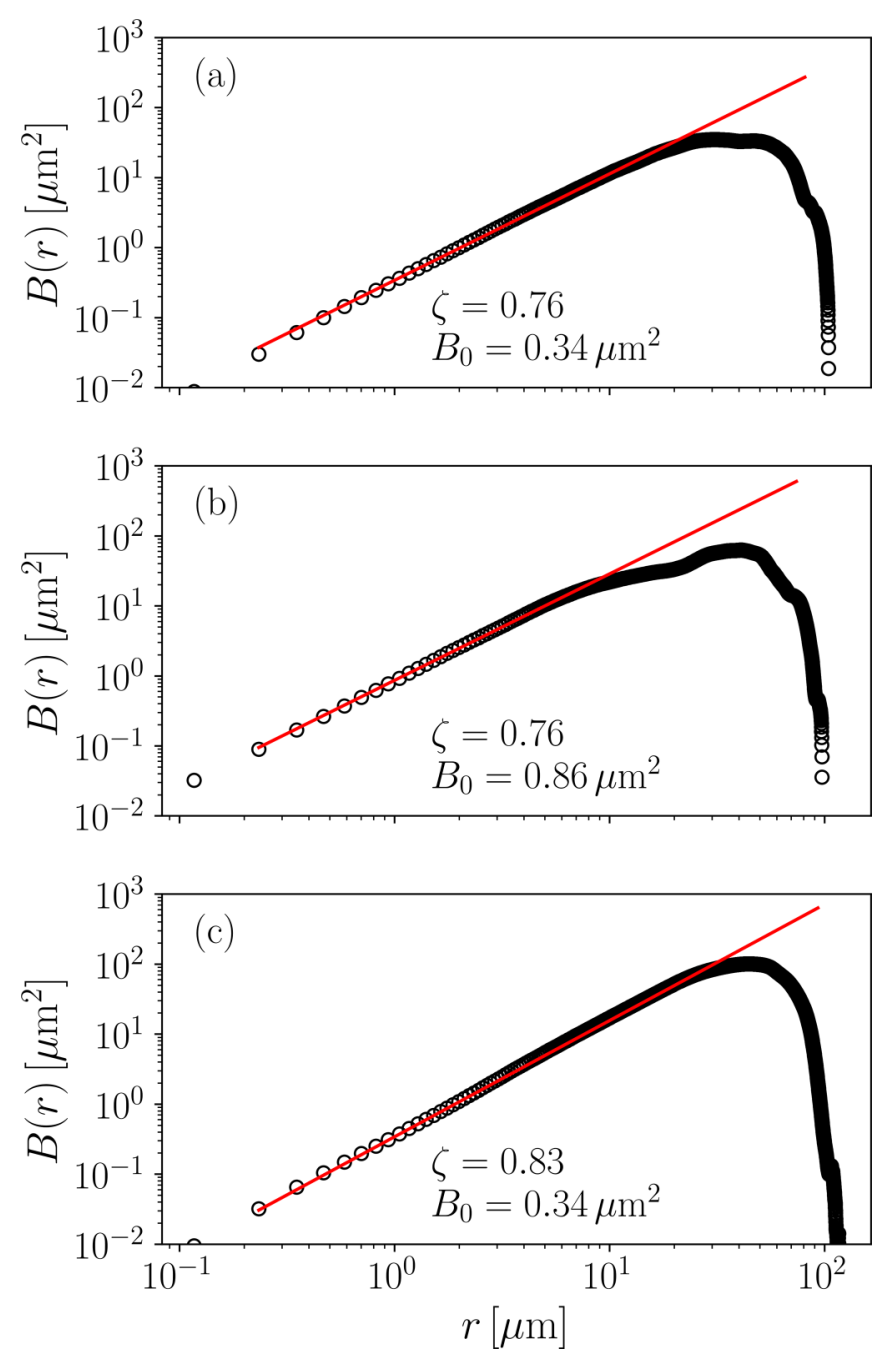

FIG. 3. Roughness function $B(r)$ corresponding to the domainwall profiles in Fig. 2. Data are represented in log-log scale and from a linear fit the roughness exponent $(\zeta)$ and roughness amplitude $\left(B_{0}\right)$ are extracted, as indicated in the keys.

It is worth noting that from a direct comparison among the profiles shown in Fig. 2 it is a priori difficult to assert whether the roughness amplitude and/or the roughness exponent are changing. Figure 3(a) presents the roughness function corresponding to Fig. 2(a) in a log-log representation. By fitting the results using the power law Eq. (5), i.e., fitting the linear regime in the log-log representation, the obtained values for the roughness exponent and roughness amplitude are $\zeta=0.76$ and $B_{0}=0.34 \mu \mathrm{m}^{2}$, respectively. Notice that while the first data point in the roughness functions shown in Fig. 3 corresponds to the pixel size (equal to $0.117 \mu \mathrm{m}$ ), the lower bound of the fit range has been set to $0.819 \mu \mathrm{m}$. Further details on the procedure followed to obtain the roughness parameters for a single $B(r)$ function are described in the Appendix.

As shown in Fig. 3(b), the roughness parameters corresponding to the domain wall in Fig. 2(b) are $\zeta=0.76$ and $B_{0}=0.86 \mu \mathrm{m}^{2}$. Therefore, the domain walls shown in Figs. 2(a) and 2(b) have similar roughness exponents but very different roughness amplitudes. Indeed, the fact that the domain wall in Fig. 2(b) looks rougher than that on Fig. 2(a) is accounted for by the value of the roughness amplitude and not by a change in the roughness exponent. Finally, Fig. 3(c) presents the roughness function for the domain wall shown in Fig. 2(c), with roughness parameters $\zeta=0.83$ and $B_{0}=$ $0.34 \mu \mathrm{m}^{2}$. Therefore, in this last case we obtain a value for the roughness exponent larger than those obtained for the other two domain-wall profiles but with the same roughness amplitude as in Fig. 2(a).

Figure 3 shows that when using Eq. (5) to fit the linear regime of the roughness function corresponding to domainwall profiles obtained under the same conditions (Fig. 2), some striking differences can be observed. Firstly, taking into account the obtained roughness parameters and comparing Figs. 2(a), 2(b) and 2(c), one can state that the naked eye is more likely to recognize differences in the roughness amplitude of the roughness function than in the roughness exponent: One could say that the domain-wall profile in Fig. 2(b) is rougher than in Fig. 2(a), related to its amplitude, but one could not say with the naked eye that the roughness exponent is changing between Fig. 2(c) and Fig. 2(a). Secondly, and more importantly, intrinsic stochastic variations in the values of both the roughness amplitude and the roughness exponent compel us to perform a detailed statistical study of these quantities. This variability also evidences that a single value of the roughness parameters is only representative of a given wall in a given situation but it can not be attributed to a statistical representation of the whole system. A detailed statistical analysis of roughness parameters is presented in the next section.

\section{STATISTICALLY REPRESENTATIVE ROUGHNESS PARAMETERS}

Since the roughness parameters present noticeable intrinsic fluctuations over domain-wall realizations, we address the question of how to properly report the results in order to provide statistically relevant material dependent parameters. As representative results we show in this section the statistical analysis of domain-wall roughness for the three studied cases shown in Fig. 1(a). This will permit us to compare roughness parameters obtained with and without an in-plane field.

Before directly comparing average roughness parameters, we notice that domain-wall profiles look rougher when the in-plane field is switched on, as shown in Fig. 4. We have systematically observed the same feature over many images and for different values of in-plane fields. Figure 4(a) shows a typical domain-wall profile corresponding to a velocity $v=0.50 \mu \mathrm{m} / \mathrm{s}$ obtained using an out-of-plane field $H_{z}=$ 32.0 Oe. Figure 4(b) shows a domain-wall profile for the same value of $H_{z}=32.0 \mathrm{Oe}$ as in Fig. 4(a) but with a finite value of the in-plane field $H_{x}=850 \mathrm{Oe}$, corresponding to $v=3.25 \mu \mathrm{m} / \mathrm{s}$. Comparing Figs. 4(a) and 4(b), a change on the roughness of the domain-wall profile is appreciable, suggesting that domain walls look rougher when the in-plane field is applied.

The main results of the statistical analysis are presented in Fig. 5. Panels (a) and (b) show the frequency histograms for the roughness exponent and the roughness amplitude for $H_{z}=32.0$ Oe and zero $H_{x}$. On one hand, taking into account 
(a)

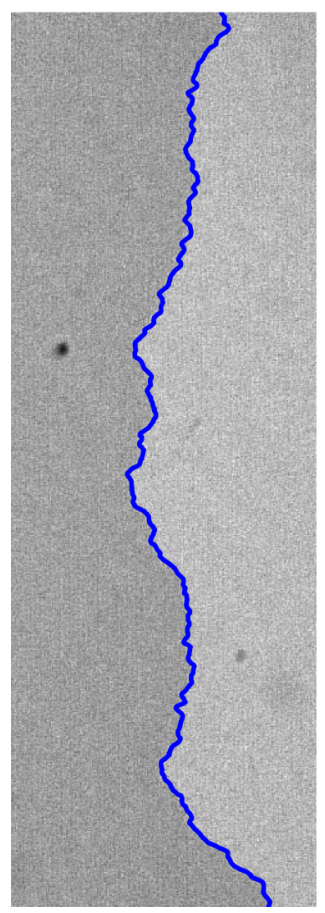

(b)

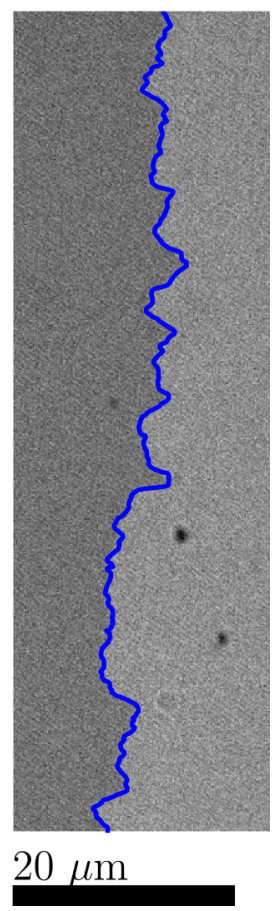

FIG. 4. Typical domain-wall profiles obtained with an out-ofplane field $H_{z}=32.0 \mathrm{Oe}$ and with zero in-plane field (a) and $H_{x}=$ 850 Oe in-plane field (b). These images show that domain walls typically look rougher when an in-plane field is applied. The propagation direction before the image acquisition was from left to right.

that it is expected that $0<\zeta<1$, since the $B(r)$ function is used to obtain the roughness exponent [37,46], the distribution of $\zeta$ values is rather wide. The histogram in Fig. 5(a) has a mean value of $\bar{\zeta}=0.759$ and a standard deviation $S_{\zeta}=0.06$, as indicated in the figure. On the other hand, the histogram of $B_{0}$ [Fig. 5(b)], which is in principle unbounded from above, presents an appreciable skewness, $\overline{B_{0}}=0.48 \mu \mathrm{m}^{2}$, and a standard deviation $S_{B}=0.2 \mu \mathrm{m}^{2}$. The results for $H_{z}=$ 32.0 Oe and a finite in-plane field $H_{x}=850 \mathrm{Oe}$ are presented in Figs. 5(c) and 5(d). It corresponds to a larger velocity than for the same $H_{z}$ and zero $H_{x}$ [Figs. 5(a) and 5(b)], and an apparently rougher domain-wall profile, as shown in Fig. 4. Finally, Figs. 5(e) and 5(f) present the distribution of $\zeta$ and $B_{0}$ for $H_{z}=35.6 \mathrm{Oe}$ and zero $H_{x}$, which corresponds to approximately the same velocity as the case with finite $H_{x}$. All cases present wide distributions with similar values for the standard deviations, as indicated in the figure, and with a noticeable skewness in the case of the roughness amplitude.

Histograms for both the roughness exponent and the roughness amplitude are not expected to tend to a Gaussian shape. On one hand, by construction the roughness function $\mathrm{B}(\mathrm{r})$ provides roughness exponents such that $0<\zeta<1$, and then its distribution is bounded and not expected to be symmetrical. On the other hand, the roughness amplitude is defined positive and then its distribution can be anticipated to have a finite skewness if the average value and the standard deviation are of the same order. Nevertheless, for a sufficiently large number of events the Gaussian shape seems to be a good approxima-

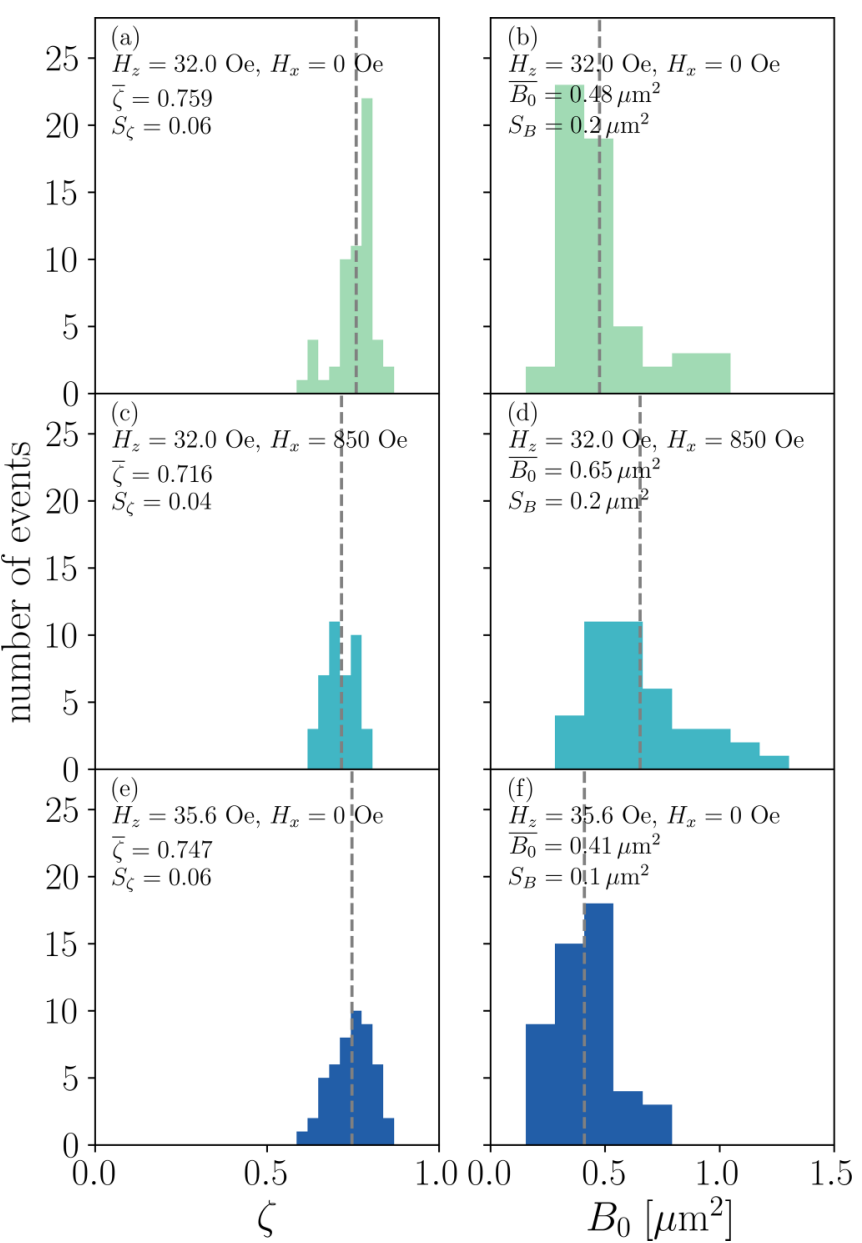

FIG. 5. Frequency histograms for the roughness exponent $\zeta$ and roughness amplitude $B_{0}$. (a),(b) correspond to $H_{z}=32.0$ Oe and $H_{x}=0$, extracted from $N=57$ domain walls. (c),(d) were obtained from $N=41$ domain walls imaged with $H_{z}=32.0$ Oe and $H_{x}=$ 850.0 Oe. (e),(f) correspond to $H_{z}=35.6$ Oe and $H_{x}=0$, obtained from $N=49$ domain walls. Average values and standard deviations are indicated for each case. Dashed vertical lines highlight how the average values are changing in each case.

tion, as shown for numerical simulations of interfaces with $N=10000$ [37]. Moreover, notice that increasing the number of domain-wall profiles would smooth the distributions but would not make them narrower.

The overall results are presented in Table I, where the average roughness parameters are indicated. This permits us to compare the obtained values and assert what is the effect of

TABLE I. Average roughness exponents and roughness amplitudes. Uncertainties account for the contributions of the standard error, coming from the standard deviations of the frequency histograms in Fig. 5, and the propagation of the individual uncertainty values (see Appendix for details).

\begin{tabular}{lcccccc}
\hline \hline$H_{z}(\mathrm{Oe})$ & $H_{x}(\mathrm{Oe})$ & $v(\mu \mathrm{m} / \mathrm{s})$ & $\bar{\zeta}$ & $\Delta \bar{\zeta}$ & $\overline{B_{0}}\left(\mu \mathrm{m}^{2}\right)$ & $\Delta \overline{B_{0}}\left(\mu \mathrm{m}^{2}\right)$ \\
\hline 32.0 & 0 & 0.50 & 0.759 & 0.008 & 0.48 & 0.02 \\
32.0 & 850 & 3.25 & 0.716 & 0.007 & 0.65 & 0.03 \\
35.6 & 0 & 3.35 & 0.747 & 0.009 & 0.41 & 0.02 \\
\hline \hline
\end{tabular}


the in-plane field in the domain-wall roughness. The presented uncertainty values, $\Delta \bar{\zeta}$ and $\Delta \overline{B_{0}}$, consider both the standard deviation of the frequency histograms and the propagation of uncertainties of each individual roughness parameter. The discussion about the evaluation of uncertainties is presented in the Appendix. A first comparison of the data in the table reveals that the values of the roughness exponents are all close to 0.75 when the in-plane field is not applied, but it is slightly smaller when $H_{x}>0$. These values have to be compared with values obtained for $\mathrm{Pt} / \mathrm{Co} / \mathrm{Pt}$, which are typically close to $0.66[9,27,30]$, even though larger values, $\zeta>0.7$, were also reported $[31,33,36]$. However, as we stated in the Introduction, the measured roughness exponent depends on the accessible experimental length scale and the (field dependent) optimal length scale of the system, $L_{\mathrm{opt}}(H)$, associated to thermal activation over typical energy barriers [26,29]. We obtain here an exponent larger than the equilibrium roughness exponent $\zeta_{\text {eq }}=2 / 3$, the expected value at length scales below $L_{\text {opt }}(H)$. In addition, the obtained value is smaller than the value $\zeta_{\text {dep }}=1.25$ predicted to be observed above $L_{\mathrm{opt}}(H)$ [26]. Further theoretical research is needed in order to rationalize the obtained values $\zeta \approx 0.75$ and $\zeta \approx 0.72$ reported here.

The pioneering report of Lemerle and coworkers [27] obtained an average roughness exponent $\bar{\zeta}=0.69 \pm 0.07$ for a $\mathrm{Pt} / \mathrm{Co} / \mathrm{Pt}$ sample in the creep regime with a reasonable statistical analysis, using $N=36$ domain walls. This value of the roughness exponent seems to compare well with the results presented in Table I. Considering the width of the roughness exponent distribution, it is important to mention that measuring the roughness exponent for a single domain wall is not enough to discuss the proper universality class.

Regarding the effect of the in-plane field, we observe in Table I that both roughness parameters seem to be affected. The roughness exponent is slightly lower (around a 5\%) while the roughness amplitude is noticeably large (more than 35\%). Therefore, when comparing domain-wall profiles as the ones presented in Fig. 4, the apparent increase of the roughness is mainly due to a statistical increase in the roughness amplitude. This can be rationalized by considering that an applied inplane field affects both the domain-wall elastic energy and the domain-wall width $[43,44,47]$. When $H_{x}>H_{\text {DMI }}$, as in the case studied here, the in-plane field compensates the internal field due to the Dzyaloshinskii-Moriya interaction and while the elastic energy decreases with $H_{x}$ the domain-wall width increases with $H_{x}$ [47]. However, it is only the domain-wall elastic energy which enters the prefactor of the roughness function, with $B_{0}$ decreasing when the elastic energy increases $[48,49]$. Hence, a decrease of the domain-wall elastic energy due to a finite $H_{x}$ value results in an increase of the roughness amplitude, as observed in our experimental results.

Finally, we notice that the number of domain-wall profiles used in each case is $N>40$, which was recently suggested to be a good lower limit to reach convergence of the mean value for the roughness exponent [37]. However, the used values seem to still be small regarding the convergence of the histogram shape. Nonetheless, the used values of $N$ are enough to obtain an estimation of the histograms widths.

\section{SUMMARY}

In summary, we have described in the present work all the steps to obtain a statistically meaningful set of roughness parameters. We discussed not only how to measure the roughness exponent $\zeta$ but we also unveil how relevant it is to report measurements of the roughness amplitude: Rougher domain-wall profiles can be generally associated with a larger value for the roughness amplitude rather than with a change in the roughness exponent.

The main result of the present work is that distributions for the roughness parameters are considerably wide, which is a signature of large fluctuations. For example, a single domainwall profile can have a roughness exponent close to 0.65 (see Fig. 5) or 0.85 within the same experimental conditions, seemingly far away from the average 0.75 value. Therefore, as already mentioned in Ref. [37], a large number of domainwall profiles should be used in the future when reporting roughness parameters. This statistical approach is important in order to discuss how mean values of the roughness parameters are affected by experimental control parameters. Interestingly, when applying an in-plane field the changes in the mean roughness parameters can be identified even though the distributions are wide. In addition, in order to compare with theoretical arguments and deepen on the understanding of domain-wall velocity-force response, it would be interesting to conduct a thorough study of how the roughness parameters evolve when changing both out-of-plane and in-plane magnetic fields.

\section{ACKNOWLEDGMENTS}

The authors acknowledge fruitful discussions with $\mathrm{M}$. Granada, V. Jeudy, A. B. Kolton, and R. G. Pregliasco. This work was partly supported by Grant No. PICT20160069/FONCyT, No. PICT2016-0906/FONCyT, and No. UNCuyo 06/C490, from Argentina.

\section{APPENDIX: MEASURING ROUGHNESS PARAMETERS AND EVALUATION OF THEIR UNCERTAINTIES}

Roughness parameters should be measured for a large number of domain walls and based on a fitting procedure which should not depend on the observer. With this aim, we present here details regarding the evaluation of uncertainty values accompanying the determination of the roughness parameters $\zeta$ and $B_{0}$, which are relevant to provide reliable statistical measures. In the following we shall consider two components of uncertainties as described in Refs. [50,51]: Type A uncertainties are based on valid statistical methods for treating data while type B have not statistical origin and are based on scientific judgment using relevant available information.

\section{Uncertainties for a single domain-wall profile}

Figure 6 shows the roughness function $B(r)$ in $\log -\log$ scale corresponding to the domain-wall profile shown in Fig. 2(a). We linearly fit the roughness function in log-log scale following Eq. (5). This forces us to define a fitting 


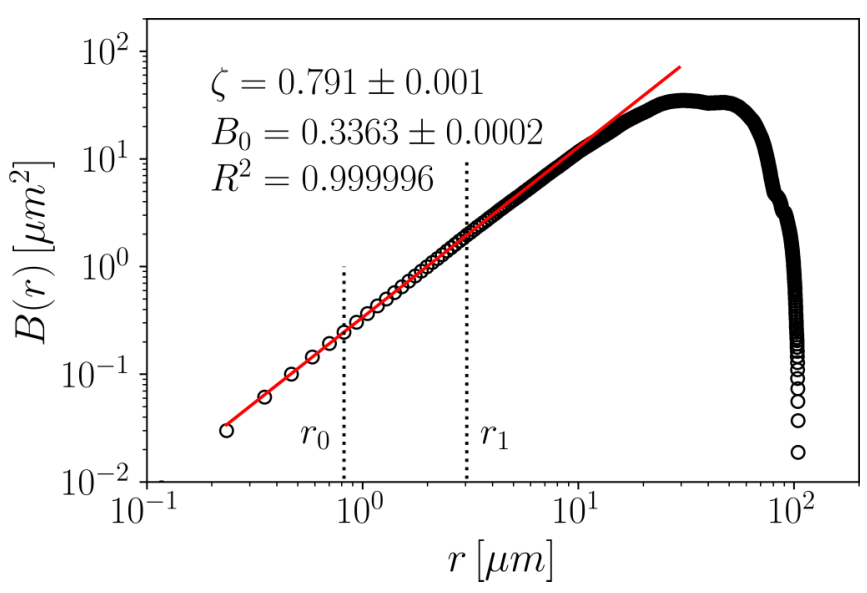

FIG. 6. Roughness function corresponding to a domain-wall profile obtained for $H_{z}=32$ Oe and $H_{x}=0$ (same data as in Fig. 3). The straight line is a linear fit according to Eq. (5), between $r_{0}$ and $r_{1}$, giving the indicated roughness parameters $\zeta$ and $B_{0}$, and the goodness of the fit $R^{2}$.

range. At first, the observer might fix the boundaries of the fitting range to $r_{0}$ and $r_{1}$ according to his/her scientific training and experience working in the subject. The lower bound is set to $r_{0}=0.819 \mu \mathrm{m}$ (corresponding to the sixth pixel of images acquired with the PMOKE setup) since for $r<r_{0}$ we typically observe deviations from the linear behavior that can be attributed to resolution effects. The upper limit $r_{1}$ of the fitting range should reflect the fact that the linear behavior is lost for larger $r>r_{1}$. The linear fit gives the roughness parameters shown in the figure. The uncertainties of these quantities are of type A [50,51] and are given by the statistical analysis of the fit. The goodness of the fit is given by the coefficient of determination $R^{2}$, indicated in the key. The roughness parameters and their uncertainties are very sensitive to the choice of $r_{1}$.

In addition, the goodness of the fit depends on the choice made for the boundary values $r_{0}$ and $r_{1}$. One expects for instance that increasing the value of $r_{1}$ the value of $R^{2}$ would eventually rapidly decay, indicating that the linear model is no longer valid. Figure 7 shows how $R^{2}$ changes with the boundaries of the fit. Blue squares show the evolution of $R^{2}$ with $r_{\max }$ when fitting between $r_{0}$ and $r_{\max }$. The dependence of $R^{2}$ with $r_{\text {min }}$ when the fitting range is $r_{\text {min }} \leqslant r \leqslant r_{1}$ is shown as green diamonds. As a phenomenological criterion we state that the goodness of the fit is poor when $R^{2}$ drops below $R^{2 *}$, with $r_{\max }$ and $r_{\min }$ reaching the values $r_{1}^{*}$ and $r_{0}^{*}$, respectively. These values are indicated in Fig. 7 for $R^{2 *}=0.9998$. Since the value of $r_{0}$ has been chosen based on the resolution of the experimental setup, it makes no sense to keep a value of $r_{0}^{*}<r_{0}$. Then we reset the value of $r_{0}^{*}$ to $r_{0}^{*}=\max \left(r_{0}, r_{0}^{*}\right)$. Of course, the obtained values for $r_{0}^{*}$ and $r_{1}^{*}$ depend on the choice of $R^{2 *}$. We shall come back to the dependence on $R^{2 *}$ below.

One possible way to move forward is to fit the data in the range $r_{0}^{*} \leqslant r \leqslant r_{1}^{*}$. This would give a value for the roughness exponent $\zeta=0.756 \pm 0.003$. However, we are also interested in reflecting how the roughness exponent varies with the chosen fitting range. This prompts us to evaluate type B uncertainties [50,51]. Therefore, Fig. 8 presents the dependence of

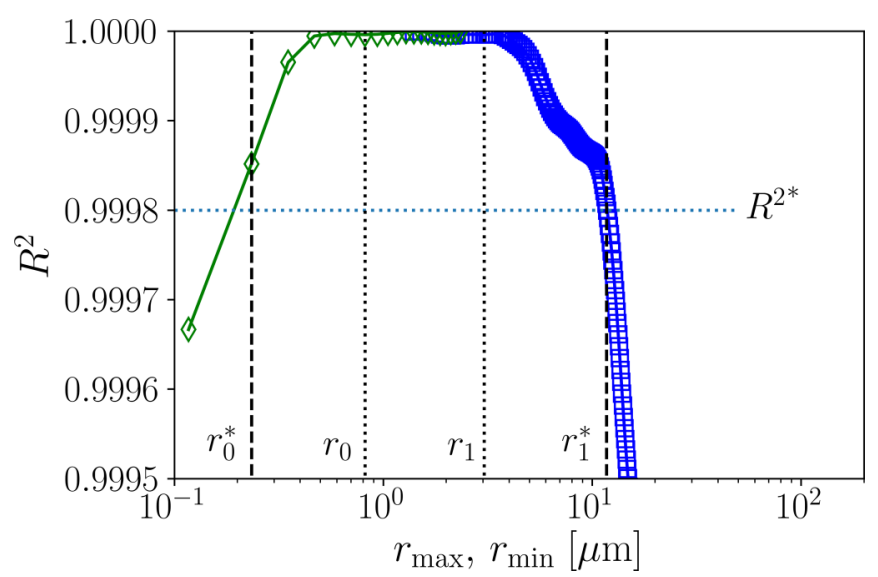

FIG. 7. Dependence of the goodness of the linear fit, $R^{2}$, on the limits of the fitting range. Blue squares correspond to fitting in the range $r_{0} \leqslant r \leqslant r_{\max }$, while green diamonds represent the result when fitting between $r_{\min }$ and $r_{1}$.

the roughness exponent on the fitting range (as shown for $R^{2}$ in Fig. 7). Blue squares and green diamonds correspond to fits in the ranges $r_{0}^{*} \leqslant r \leqslant r_{\max }$ and $r_{\min } \leqslant r \leqslant r_{1}^{*}$, respectively. The values for $r_{0}^{*}$ and $r_{1}^{*}$ shown in Fig. 8 correspond to those obtained in Fig. 7. The value of $\zeta$ obtained when fitting in the range $r_{0}^{*} \leqslant r \leqslant r_{1}^{*}$ is the one corresponding to the blue square exactly at $r_{1}^{*}$ in Fig. 8. For this example, the value of $\zeta=0.756$ is the lower bound of all the possible values of $\zeta$ when varying the fitting range. Hence, variations of $\zeta$ due to changes in the fitting range are certainly outside the statistical uncertainty value of a single fit. We then report for $\zeta$ what we consider the most representative value for the roughness exponent, defined as

$$
\zeta=\left[\max \left(\zeta_{\mathrm{fit}}\right)+\min \left(\zeta_{\mathrm{fit}}\right)\right] / 2,
$$

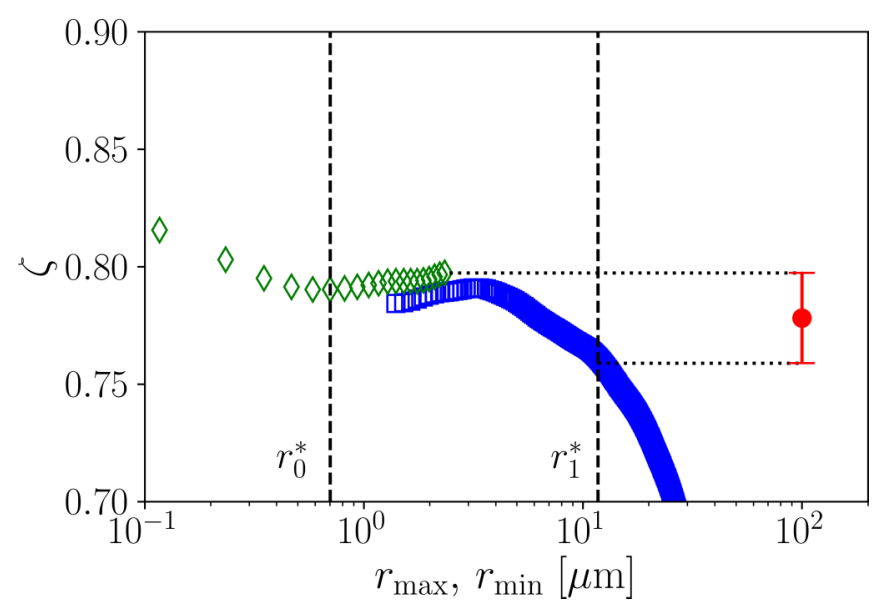

FIG. 8. Dependence of the roughness exponent parameter on the limits of the fitting range. Blue squares correspond to fitting in the range $r_{0}^{*} \leqslant r \leqslant r_{\max }$ while green diamonds represent the result when fitting between $r_{\min }$ and $r_{1}^{*}$. The vertical dashed lines correspond to the values of $r_{0}^{*}$ and $r_{1}^{*}$ obtained in Fig. 7. The representative value obtained using Eqs. (A1) and (A2) is shown as a red point. 


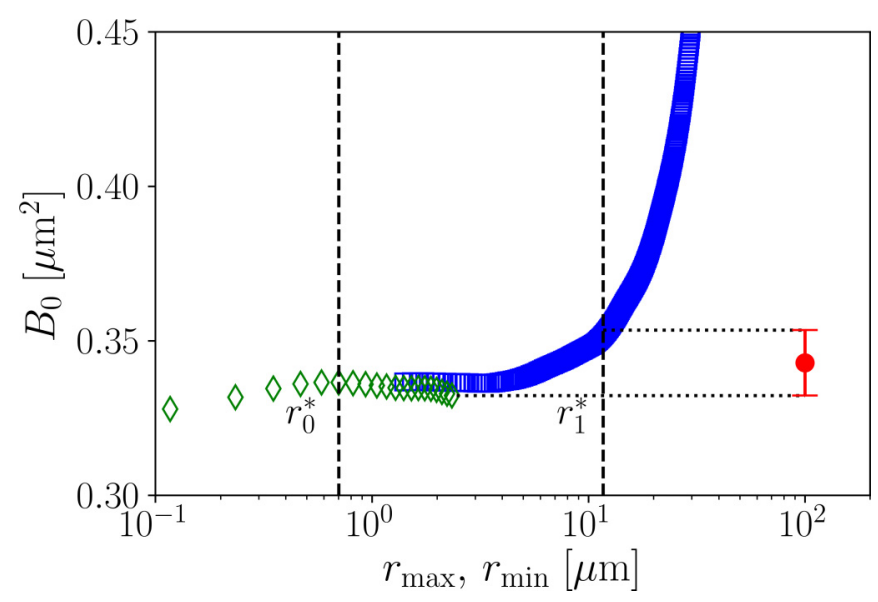

FIG. 9. Dependence of the roughness amplitude parameter on the limits of the fitting range. Blue squares correspond to fitting in the range $r_{0}^{*} \leqslant r \leqslant r_{\max }$ while green diamonds represent the result when fitting between $r_{\min }$ and $r_{1}^{*}$. The vertical dashed lines correspond to the values of $r_{0}^{*}$ and $r_{1}^{*}$ obtained in Fig. 7. The representative value obtained using Eqs. (A3) and (A4) is shown as a red point.

with its corresponding uncertainty

$$
\Delta \zeta=\left[\max \left(\zeta_{\text {fit }}\right)-\min \left(\zeta_{\text {fit }}\right)\right] / 2,
$$

where $\zeta_{\text {fit }}$ contains all possible $\zeta$ values in the range $r_{0}^{*} \leqslant r \leqslant$ $r_{1}^{*}$, i.e., those between the vertical dashed lines in Fig. 8. The procedure is indicated in Fig. 8 where the horizontal dotted lines correspond to the maximum and minimum of $\zeta_{\text {fit }}$ and the red dot represents the final result $\zeta \pm \Delta \zeta=0.78 \pm 0.02$.

Once the values of $r_{0}^{*}$ and $r_{1}^{*}$ are fixed following the previously described procedure, the corresponding value for the roughness amplitude can also be obtained as for the roughness exponent in Fig. 8. Figure 9 shows the values for the roughness amplitude depending on the fitting range. As for the case of the roughness exponent we obtain a representative value for the roughness amplitude from

$$
\begin{gathered}
B_{0}=\left[\max \left(B_{0 \text { fit }}\right)+\min \left(B_{0 \text { fit }}\right)\right] / 2, \\
\Delta B_{0}=\left[\max \left(B_{0 \text { fit }}\right)-\min \left(B_{0 \text { fit }}\right)\right] / 2,
\end{gathered}
$$

which contains information on how it changes with the fitting range in the same range as for the roughness exponent. The obtained value is $B_{0} \pm \Delta B_{0}=0.34 \mu \mathrm{m}^{2} \pm 0.01 \mu \mathrm{m}^{2}$ and it is indicated in Fig. 9 as a red point.

The values thus obtained for the roughness parameters and their uncertainties would depend on the choice of the threshold value for the goodness of the fit, $R^{2 *}$. This choice depends on the quality of the original experimental data set and the expected accuracy. In fact, domain-wall profiles whose $B(r)$ is too noisy or presents an appreciable curvature will have a goodness below $R^{2 *}$ and would be eliminated from the data set. Therefore, a very large value of $R^{2 *}$ would eliminate a large part of the domain-wall profiles, reducing statistical average, while a small value of $R^{2 *}$ would increase the un- certainty value of the measured roughness parameters, and might result in considering domain-wall profiles for which the linear model would not be a good description. We took here the value $R^{2 *}=0.9998$ which permits us to have a large data set on each case $(N>40)$ with good accuracy for the reported results.

\section{Uncertainties for the whole data set}

Now, we move forward to obtain uncertainty values for the roughness parameters for the whole data set. Let us consider a set of $N$ domain-wall profiles obtained for given experimental conditions. Domain wall profiles are labeled with index $i=1,2, \ldots, N$. This could be for instance any of the three data sets discussed in this work. Then, for each profile the roughness function $B_{i}(r)$ is computed. Using the procedure just described, the roughness exponent and the roughness amplitude are obtained, $\zeta_{i}$ and $B_{0 i}$, with their corresponding uncertainties, $\Delta \zeta_{i}$ and $\Delta B_{0 i}$.

The set of $N$ values for $\zeta_{i}$ and $B_{0 i}$ serve for building the histograms shown in Fig. 5 and for computing their average values $\bar{\zeta}=N^{-1} \sum_{i} \zeta_{i}$ and $\overline{B_{0}}=N^{-1} \sum_{i} B_{0 i}$, as reported in Table I. There are two possible origins for the uncertainty of the average value. One is of direct statistical origin and accounts for the fact that roughness exponents are distributed according to a given distribution, reflected by the shown histograms. In this case, for the roughness exponent, the uncertainty is given by $\Delta \bar{\zeta}^{\text {std }}=S_{\zeta} / \sqrt{N}$, where $S_{\zeta}$ is the standard deviation of the data. The other origin is the propagation of the individual uncertainty values $\Delta \zeta_{i}$ to the uncertainty of the average. This results in $\Delta \bar{\zeta}^{\text {avg }}=\sqrt{N^{-2} \sum_{i}\left(\Delta \zeta_{i}\right)^{2}}$. Therefore, considering both cases, the uncertainty for the average roughness exponent is given by

$$
\Delta \bar{\zeta}=\sqrt{\left(\Delta \bar{\zeta}^{\text {std }}\right)^{2}+\left(\Delta \bar{\zeta}^{\text {avg }}\right)^{2}}
$$

Similarly for the roughness amplitude, we get $\Delta{\overline{B_{0}}}^{\text {std }}=$ $S_{B} / \sqrt{N}$ and $\Delta \overline{B_{0}}{ }^{\text {avg }}=\sqrt{N^{-2} \sum_{i}\left(\Delta B_{0 i}\right)^{2}}$, and the uncertainty given thus by

$$
\Delta \overline{B_{0}}=\sqrt{\left(\Delta{\overline{B_{0}}}^{\text {std }}\right)^{2}+\left(\Delta{\overline{B_{0}}}^{\text {avg }}\right)^{2}} .
$$

This is how the reported uncertainties values in Table I were computed.

In our case, the uncertainties for $\bar{\zeta}$ and $\overline{B_{0}}$ are mainly given by the standard deviation of the distributions, whose contribution is always larger than the uncertainty coming from propagating individual uncertainty values. For example, for the case with $H_{z}=32.0$ Oe and $H_{x}=0$, we obtained $\Delta \bar{\zeta}^{\text {std }}=$ 0.007 and $\Delta \bar{\zeta}^{\text {avg }}=0.003$, resulting in the uncertainty value $\Delta \bar{\zeta}=0.008$ reported in Table I. Of course, which uncertainty contributes more to the final value would depend on the quality of the obtained domain-wall profiles and on the number of individual profiles within a data set. That is the reason why it is necessary to properly account for individual uncertainty values.
[1] D. A. Allwood, G. Xiong, C. C. Faulkner, D. Atkinson, D. Petit, and R. P. Cowburn, Science 309, 1688 (2005).
[2] M. Hayashi, L. Thomas, R. Moriya, C. Rettner, and S. S. P. Parkin, Science 320, 209 (2008). 
[3] S. S. P. Parkin, M. Hayashi, and L. Thomas, Science 320, 190 (2008).

[4] R. L. Stamps, S. Breitkreutz, J. Åkerman, A. V. Chumak, Y. Otani, G. E. W. Bauer, J.-U. Thiele, M. Bowen, S. A. Majetich, M. Kläui, I. L. Prejbeanu, B. Dieny, N. M. Dempsey, and B. Hillebrands, J. Phys. D: Appl. Phys. 47, 333001 (2014).

[5] F. Hellman, A. Hoffmann, Y. Tserkovnyak, G. S. D. Beach, E. E. Fullerton, C. Leighton, A. H. MacDonald, D. C. Ralph, D. A. Arena, H. A. Dürr, P. Fischer, J. Grollier, J. P. Heremans, T. Jungwirth, A. V. Kimel, B. Koopmans, I. N. Krivorotov, S. J. May, A. K. Petford-Long, J. M. Rondinelli, N. Samarth, I. K. Schuller, A. N. Slavin, M. D. Stiles, O. Tchernyshyov, A. Thiaville, and B. L. Zink, Rev. Mod. Phys. 89, 025006 (2017).

[6] D. Sander, S. O. Valenzuela, D. Makarov, C. H. Marrows, E. E. Fullerton, P. Fisher, J. McCord, P. Vavassori, S. Mangin, P. Pirro, B. Hillebrands, A. D. Kent, T. Jungwirth, O. Gutfleisch, C. G. Kim, and A. Berger, J. Phys. D: Appl. Phys. 50, 363001 (2017).

[7] F. Ummelen, H. Swagten, and B. Koopmans, Sci. Rep. 7, 833 (2017).

[8] J. Curiale, A. Lemaître, C. Ulysse, G. Faini, and V. Jeudy, Phys. Rev. Lett. 108, 076604 (2012).

[9] K.-W. Moon, D.-H. Kim, S.-C. Yoo, C.-G. Cho, S. Hwang, B. Kahng, B.-C. Min, K.-H. Shin, and S.-B. Choe, Phys. Rev. Lett. 110, 107203 (2013).

[10] S. DuttaGupta, S. Fukami, C. Zhang, H. Sato, M. Yamanouchi, F. Matsukura, and H. Ohno, Nat. Phys. 12, 333 (2016).

[11] K.-W. Moon, C. Kim, J. Yoon, J. W. Choi, D.-O. Kim, K. M. Song, D. Kim, B. S. Chun, and H. Hwang, Nat. Commun. 9, 3788 (2018).

[12] A. Schellekens, A. van den Brink, J. Franken, H. Swagten, and B. Koopmans, Nat. Commun. 3, 847 (2012).

[13] D. Chiba, M. Kawaguchi, S. Fukami, N. Ishiwata, K. Shimamura, K. Kobayashi, and T. Ono, Nat. Commun. 3, 888 (2012).

[14] A. Bernand-Mantel, L. Herrera-Diez, L. Ranno, S. Pizzini, J. Vogel, D. Givord, S. Auffret, O. Boulle, I. M. Miron, and G. Gaudin, Appl. Phys. Lett. 102, 122406 (2013).

[15] T. Koyama, Y. Nakatani, J. Ieda, and D. Chiba, Sci. Adv. 4, eaav0265 (2018).

[16] P. M. Shepley, A. W. Rushforth, M. Wang, G. Burnell, and T. A. Moore, Sci. Rep. 5, 792 (2015).

[17] P. M. Shepley, G. Burnell, and T. A. Moore, J. Phys. Condens. Matter 30, 344002 (2018).

[18] S.-G. Je, D.-H. Kim, S.-C. Yoo, B.-C. Min, K.-J. Lee, and S.-B. Choe, Phys. Rev. B 88, 214401 (2013).

[19] A. Hrabec, N. A. Porter, A. Wells, M. J. Benitez, G. Burnell, S. McVitie, D. McGrouther, T. A. Moore, and C. H. Marrows, Phys. Rev. B 90, 020402(R) (2014).

[20] R. Lavrijsen, D. M. F. Hartmann, A. van den Brink, Y. Yin, B. Barcones, R. A. Duine, M. A. Verheijen, H. J. M. Swagten, and B. Koopmans, Phys. Rev. B 91, 104414 (2015).

[21] M. Vaňatka, J.-C. Rojas-Sánchez, J. Vogel, M. Bonfim, M. Belmeguenai, Y. Roussigné, A. Stashkevich, A. Thiaville, and S. Pizzini, J. Phys.: Condens. Matter 27, 326002 (2015).
[22] A. W. J. Wells, P. M. Shepley, C. H. Marrows, and T. A. Moore, Phys. Rev. B 95, 054428 (2017).

[23] D.-Y. Kim, M.-H. Park, Y.-K. Park, J.-S. Kim, Y.-S. Nam, H.-S. Hwang, D.-H. Kim, S.-G. Je, B.-C. Min, and S.-B. Choe, Phys. Rev. B 97, 134407 (2018).

[24] M. V. Feigel'man, V. B. Geshkenbein, A. I. Larkin, and V. M. Vinokur, Phys. Rev. Lett. 63, 2303 (1989).

[25] T. Nattermann, Phys. Rev. Lett. 64, 2454 (1990).

[26] A. B. Kolton, A. Rosso, T. Giamarchi, and W. Krauth, Phys. Rev. Lett. 97, 057001 (2006).

[27] S. Lemerle, J. Ferré, C. Chappert, V. Mathet, T. Giamarchi, and P. Le Doussal, Phys. Rev. Lett. 80, 849 (1998).

[28] E. E. Ferrero, S. Bustingorry, A. B. Kolton, and A. Rosso, C. R. Physique 14, 641 (2013).

[29] M. P. Grassi, A. B. Kolton, V. Jeudy, A. Mougin, S. Bustingorry, and J. Curiale, Phys. Rev. B 98, 224201 (2018).

[30] T. Shibauchi, L. Krusin-Elbaum, V. M. Vinokur, B. Argyle, D. Weller, and B. D. Terris, Phys. Rev. Lett. 87, 267201 (2001).

[31] M. Huth, P. Haiback, and H. Adrian, J. Magn. Magn. Mater. 240, 311 (2002).

[32] M. Bauer, A. Mougin, J. P. Jamet, V. Repain, J. Ferré, R. L. Stamps, H. Bernas, and C. Chappert, Phys. Rev. Lett. 94, 207211 (2005).

[33] K.-S. Lee, C.-W. Lee, Y.-J. Cho, S. Seo, D.-H. Kim, and S.-B. Choe, IEEE Trans. Magn. 45, 2548 (2009).

[34] W. S. Torres, R. D. Pardo, S. Bustingorry, A. B. Kolton, A. Lemaître, and V. Jeudy, Phys. Rev. B 99, 201201(R) (2019).

[35] R. Diaz Pardo, N. Moisan, L. Albornoz, A. Lemaître, J. Curiale, and V. Jeudy, Phys. Rev. B 100, 184420 (2019).

[36] P. Domenichini, C. P. Quinteros, M. Granada, S. Collin, J.-M. George, J. Curiale, S. Bustingorry, M. G. Capeluto, and G. Pasquini, Phys. Rev. B 99, 214401 (2019).

[37] J. Guyonnet, E. Agoritsas, P. Paruch, and S. Bustingorry, arXiv:1904.11726.

[38] P. Hansen, C. Clausen, G. Much, M. Rosenkranz, and K. Witter, J. Appl. Phys. 66, 756 (1989).

[39] K.-J. Kim, S. K. Kim, Y. Hirata, S.-H. Oh, T. Tono, D.-H. Kim, T. Okuno, W. S. Ham, S. Kim, G. Go, Y. Tserkovnyak, A. Tsukamoto, T. Moriyama, K.-J. Lee, and T. Ono, Nature Mat. 16, 1187 (2017).

[40] Y. Hirata, D.-H. Kim, T. Okuno, T. Nishimura, D.-Y. Kim, Y. Futakawa, H. Yoshikawa, A. Tsukamoto, K.-J. Kim, S.-B. Choe, and T. Ono, Phys. Rev. B 97, 220403(R) (2018).

[41] V. Jeudy, A. Mougin, S. Bustingorry, W. Savero Torres, J. Gorchon, A. B. Kolton, A. Lemaître, and J.-P. Jamet, Phys. Rev. Lett. 117, 057201 (2016).

[42] V. Jeudy, R. Diaz Pardo, W. Savero Torres, S. Bustingorry, and A. B. Kolton, Phys. Rev. B 98, 054406 (2018).

[43] J. P. Pellegren, D. Lau, and V. Sokalski, Phys. Rev. Lett. 119, 027203 (2017).

[44] E. Jué, A. Thiaville, S. Pizzini, J. Miltat, J. Sampaio, L. D. Buda-Prejbeanu, S. Rohart, J. Vogel, M. Bonfim, O. Boulle, S. Auffret, I. M. Miron, and G. Gaudin, Phys. Rev. B 93, 014403 (2016).

[45] A.-L. Barabási and H. E. Stanley, Fractal Concepts in Surface Growth (Cambridge University Press, Cambridge, 1995). 
[46] J. M. López, M. A. Rodríguez, and R. Cuerno, Phys. Rev. E 56, 3993 (1997).

[47] D.-Y. Kim and D.-H. K. S.-B. Choe, App. Phys. Express 9, 053001 (2016).

[48] E. Agoritsas, V. Lecomte, and T. Giamarchi, Phys. Rev. B 82, 184207 (2010).
[49] E. Agoritsas, V. Lecomte, and T. Giamarchi, Physica B 407, 1725 (2012).

[50] NIST, https://physics.nist.gov/cuu/Uncertainty/index.html.

[51] N. Taylor and C. E. Kuyatt, NIST Technical Note 1297, National Institute of Standards and Technology, 1994, https://dx. doi.org/10.6028/NIST.TN.1297. 